\title{
Superconducting Superhydrides: Synthesis, Structure and Stability
}

\author{
M Somayazulu ${ }^{1}$ \\ ${ }^{1}$ HPCAT-XSD, Advanced Photon Source, Argonne National Laboratory, Lemont, USA \\ zulu@anl.gov
}

Room-temperature superconductivity was first predicted in metallic hydrogen and then postulated in a number of hydrogen-rich materials at very high pressures $/ 1,2 /$. The search for these superconductors led through hydrogen and related molecular hydrides culminating in the exciting discovery and concomitant theoretical simulations of superconductivity in $\mathrm{H}[\mathrm{sub}] 3[/ \mathrm{sub}] \mathrm{S} / 3-5 /$. The field practically exploded with this successful synergy between theory and experiment culminating in the discovery of superconductivity in $\mathrm{YH}[\mathrm{sub}] \mathrm{x}[/ \mathrm{sub}], \mathrm{LaH}[\mathrm{sub}] 10-\mathrm{x}[/ \mathrm{sub}]$ at temperatures as high as $265 \mathrm{~K} / 6-10 /$. The pressures of synthesis make these compounds (as yet) unsuitable for neutron diffraction and therefore one relies on spectroscopy and x-ray diffraction to correlate with theoretical models and hypothesize the structures $/ 8 /$. Our experiments reveal a very nebulous pathway to synthesis and stability and correlation between $\mathrm{T}[\mathrm{sub}] \mathrm{c}[/ \mathrm{sub}]$ and hydrogen stoichiometry. This talk will focus on the structure and stability aspects of these interesting class of compounds that need to be well understood to have a reproducible pathway to synthesis and validation of other properties including Meissner effect.

[b]Acknowledgements[/b]: The synchrotron x-ray diffraction measurements were carried out at 16-ID-B of HPCAT at the Advanced Photon Source, a U.S. Department of Energy (DOE) Office of Science User Facility operated for the DOE Office of Science by Argonne National Laboratory under Contract No. DE-AC0206CH11357. Funding is acknowledged from DOE-BES, DOE-NNSA and NSF-DMR.

[b]References[/b]:

/1/ N. W. Ashcroft, [i]Metallic Hydrogen: A High-Temperature Superconductor?[/i], Phys. Rev. Lett., [b]21[/b], 1748 (1968).

/2/ N. W. Ashcroft, [i]Hydrogen Dominant Metallic Alloys: High Temperature Superconductors?[/i], Phys. Rev. Lett., [b]92[/b], 187002 (2004).

/3/ A. P. Drozdov et. al, [i]Conventional Superconductivity at 203 Kelvin at High Pressures in the Sulfur Hydride System[/i], [b]525[/b], 73 (2015).

/4/ Li, Y. et. al, [i]The metallization and superconductivity of dense hydrogen sulfide.[/i], J. Chem. Phys. [b]140[/b], 174712 (2014).

/5/ Duan, D. et al. [i]Pressure-induced metallization of dense $(\mathrm{H}[\mathrm{sub}] 2[/ \mathrm{sub}] \mathrm{S})[\mathrm{sub}] 2[/ \mathrm{sub}] \mathrm{H}[\mathrm{sub}] 2[/ \mathrm{sub}]$ with high-T[sub]c[/sub] superconductivity[/i]. Sci. Rep. [b]4[/b], 6968 (2014).

/6/ Hanyu Liu et. al, [i]Potential high-Tc superconducting lanthanum and yttrium hydrides at high pressure[/i], PNAS, [b]114[/b], 6990 (2017).

/7/ P.P. Kong et. al, [i]S Superconductivity up to $243 \mathrm{~K}$ in yttrium hydrides under high pressure[/i], arXiv:1909.10482 (2019).

/8/ Somayazulu, M. et al. [i]Evidence for superconductivity above $260 \mathrm{~K}$ in lanthanum superhydride at megabar pressures[ii]. Phys. Rev. Lett. [b]122[/b], 027001 (2019).

/9/ Drozdov, A. P. et al. [i]Superconductivity at $250 \mathrm{~K}$ in lanthanum hydride under high pressures[/i]. Nature [b]569[/b], 528-531 (2019).

Acta Cryst. (2020). A76, a160 